\title{
Wider implications of video-assisted thoracic surgery versus open approach for lung metastasectomy
}

\author{
Marcello Migliore ${ }^{*, 1,2}$, Alessandra Criscione ${ }^{1,2}$, Damiano Calvo ${ }^{1,2}$, Giuseppe Privitera ${ }^{3}$, \\ Corrado Spatola ${ }^{3}$, Ector Soto Parra ${ }^{4}$, Stefano Palmucci ${ }^{5}$, Nicola Ciancio ${ }^{6}$, \\ Massimo Cajozzo $\&$ Giuseppe Di Maria ${ }^{6,8}$
}

\begin{abstract}
Lung metastasectomy is considered a safe and potentially curative procedure despite there is not a strong evidence that metastasectomy prolongs long-term survival in patients with lung metastases. Moreover, the debate is open regarding the best approach for lung metastasectomy, video-assisted thoracic surgery versus open approach. A systematic review of literature to clarify what is the best approach to prolong survival in patients with lung metastases was performed. Our study confirms that overall survival is equivalent for video-assisted thoracic surgery and thoracotomy, therefore the 'gold standard' surgical treatment for lung metastases remains a point of debate. The choice of the surgical approach still depends more on the single center or surgeon practice than on strong scientific evidence. A prospective randomized trial could clarify the question.
\end{abstract}

Lung metastasectomy is considered as a safe and potentially curative procedure despite there is not a strong evidence that metastasectomy truly prolongs long-term survival in patients with lung metastases [1].

Although in surgical practice there are several approaches to pulmonary metastasectomy (PM), the proper method to use is still controversial. Furthermore since the introduction of minimally invasive techniques, most authors prefer the classic three ports video-assisted thoracic surgery (VATS) approach, few favor the uniportal method, but many surgeons still use the standard open approach. The advantage of the open approach consists in the fact that the method permits to palpate the lung to discover additional small lung nodules. On the contrary VATS for PM remains controversial. Critics to the VATS have argued that it might not be an equivalent oncological operation by intention to cure as demonstrated by a prospective study where $22 \%$ of the nodules that could be detected by thoracotomy (TT) were missing by VATS [2]. Moreover, a recent study demonstrated that the incidence of the nodules that were not imaged pre-operatively was 36\% [3].

The question that arise is obvious. Is VATS better than TT to perform lung metastasectomy? To answer these questions we undertook a systematic review of the literature.

\footnotetext{
'Academic Thoracic Surgery Unit, A.O.U. Policlinico-Vittorio Emanuele, Catania, Italy

2Department of Surgery, University of Catania, Catania, Italy

${ }^{3}$ Radiotherapy Unit, A.O.U. Policlinico-Vittorio Emanuele, University of Catania, Catania, Italy

${ }^{4}$ Oncology Unit, A.O.U. Policlinico-Vittorio Emanuele, Catania, Italy

${ }^{5}$ Radiology Unit, A.O.U. Policlinico-Vittorio Emanuele, University of Catania, Catania, Italy

${ }^{6}$ Pneumology Unit, A.O.U. Policlinico-Vittorio Emanuele, Catania, Italy

${ }^{7}$ Thoracic Surgery Unit, University of Palermo, Palermo, Italy

${ }^{8}$ Department of Clinical \& Molecular Bio-Medicine, University of Catania, Catania, Italy

*Author for correspondence: mmiglior@unict.it
}

\section{KEYWORDS}

- gold standard • lung

- lung metastasectomy

- pulmonary metastases

- survival • thoracotomy

- VATS • video-assisted

thoracic surgery 


\section{Method}

A systematic review was conducted according to Preferred Reporting Items for Systematic Reviews and Meta-Analyses recommendations. The words search included: 'pulmonary metastasectomy', 'lung metastasectomy', 'video-assisted thoracoscopic surgery' and 'lung metastasis'. The research was limited to human and adults. We considered all published articles from 1990 to June 2014, which reported on at least sufficient data on surgical outcome, to be eligible. The literature search was limited to articles in English and to primary reports. We evaluated the reports' quality from the titles and abstracts, identified according to the predefined eligibility criteria. The full text articles of studies that potentially met the inclusion criteria were reviewed to assess their definitive eligibility. Papers were reviewed by two of the authors (A Criscione and M Migliore). Exclusion criteria: no attempt was made to locate unpublished material. Reviews and teaching articles which contributed no data for analysis were also excluded, as were case reports, small series or videos or articles on pediatric patients.

\section{Results}

We have found 806 articles. From these, two papers were meta-analysis with the intent to provide a more accurate comparison between VATS and TT for metastatic lung cancer, two paper were systematic analysis and a further nine provided evidence to answer the questions.

\section{Meta-analysis}

Two meta-analysis are available in literature. Dong et al. [4] analysed six retrospective studies with a total of 546 patients included. Two hundred and thirty-five patients were allocated to the VATS group, whereas 311 were allocated to the open TT group to evaluate their survival rate. The two groups did not demonstrate a statistically significant difference in the 1-, 3-and 5 -year survival rates, but there were significant 3 -year disease-free survival rate benefits with open TT $(p=0.04)$. Authors conclude that VATS cannot completely replace open TT, and advocate a large multicenter randomized trial comparing these surgical techniques. The paper of Herle et al. [5], that is available only in abstract form, analyzed nine studies with a total of 796 patients. The abstract does not reports survival and disease free survival rates. Authors consider VATS as a possible alternative to open TT for
PM with equivalent survival and recurrence free survival.

\section{Systematic review}

Greenwood et al. [6] analyzed seven nonrandomized retrospective studies. VATS metastasectomy has been associated with shorter hospital stay, chest drainage times and low rate of perioperative complications. Authors noticed a lack of high-quality data, which makes it impossible to recommend any particular surgical approach in terms of long-term survival, and advocate further studies. The paper of Molnar et al. [7], analyzing seven studies concluded that palpation of the lung is still necessary in a therapeutic metastasectomy as opposed to a diagnostic procedure, when VATS is adequate.

\section{Comparative studies}

Seven papers (Table 1) compare VATS versus TT for different tumor metastases (mostly colorectal and sarcoma). These studies reported in Table 1 showed no difference in 5-year survival or diseasefree survival at 3-years between the two groups. VATS shows that length of postoperative stay ( $p$ $<0.0001$ ) and duration of chest drainage were significantly lower than TT group. Chao et al. [9] found no difference between the two group in terms of overall recurrences (54\% TT vs 40\% VATS; $\mathrm{p}=0.23$ ). Nakas et al. [13] reported disease free interval of 20 months for VATS and 29 months for the TT group with a $\mathrm{p}=0.72$. Another six authors focus on missed metastases (Table 2), and 5 of them demonstrate that many lung malignant nodules would have been missed if VATS was used exclusively, and consequently VATS is considered inadequate if the intention is to resect all pulmonary metastases during surgery (Table 2). On the contrary Nakas et al. [13] conclude that the insertion of the surgical digit is not mandatory, and advice to trust the radiologist's eye.

\section{Discussion}

PM is part of the current surgical practice. The criteria for resection have been published [20] in 1995 and are the following: the primary site of disease has to be controlled, complete resection of lung disease has to be feasible, absence of extrapulmonary metastases, the patient has to be able to tolerate planned procedures and no better alternative can be available. Predictors of favorable outcomes are extended disease-free interval (DFI), limited number of lung metastases, and completeness of resection. According to 
Table 1. Papers comparing disease-free interval and overall survival between video-assisted thoracic surgery and thoracotomy for different tumor metastases.

\begin{tabular}{|c|c|c|c|c|c|c|c|c|c|c|c|c|}
\hline Year & Pts (n) & VATS (n) & TT (n) & DFS VATS\% & DFS TT & $p$-value & Significant? & OS VATS & OS TT & $p$-value & Significant? & Author \\
\hline 2009 & 60 & 31 & 29 & 26.4 & 24.8 & 0.74 & No & 70.9 & 34 & 0.2 & No & Gossot et al. [10] \\
\hline 2012 & 143 & 90 & 53 & 40 & 54 & 0.23 & No & 51 & 43 & 0.21 & No & Chao et al. [9] \\
\hline 2008 & 143 & 72 & 71 & 34.4 & 21.1 & 0.064 & No & 49.3 & 39,5 & 0.047 & Yes & Nakajima et al. [12] \\
\hline 2002 & 20 & 8 & 12 & 50 & 42 & - & - & 67 & 70 & 0.85 & No & Mutsaerts et al. [11] \\
\hline 1998 & 22 & 22 & 16 & - & - & - & - & 56.4 & 48.6 & - & No & Watanabe et al. [14] \\
\hline 2009 & 171 & 36 & 135 & 67 & 51 & 0.27 & No & 69.6 & 58.8 & 0.24 & No & Carballo et al. [8] \\
\hline 2009 & 52 & 25 & 27 & 20 & 29 & 0.72 & No & 72 & 80 & 0.75 & No & Nakas et al. [13] \\
\hline
\end{tabular}

results of analysis of the International Registry of Lung Metastases, the reported 5-year overall survival is $36 \%$ [21]. Colorectal and sarcoma PM comprise $50 \%$ of resections with a 5 -year survival of $30-68 \%$ and $23-50 \%$, respectively. The reported 5-year survival after metastasectomy for melanoma ranged from 4.5 to $38 \%$, and the 5-year survival ranges from 37 to $53 \%$ after resection of LM with renal-cell carcinoma. Lung metastases occur in 4-25\% of patients with neck and head cancers, and the reported 5-year survival 21-59\% Scanty authors report outcome after resection of LM in patients with esophageal, gastric, hepatocellular and uterine tumors [22].

There exist different techniques to perform LM such as posterolateral and anterior TT, that allows to palpate one lung, and median sternotomy, clamshell or hemiclamshell TT and bilateral TT, used when is needed to palpate both lungs. VATS can be performed using the classic threeport method, two-ports or uniportal approach [23]. Detterbeck et al. [17] and Mineo et al. [24] introduced a substernal transxiphoid approach for bilateral lung metastasectomy.

Our data confirm that there is no agreement about surgical treatment of lung metastases, and this has been also confirmed in a recent review [22]. Although it is evident that TT permits to palpate extra nodules, benefit of survival is not obvious and consequently doubts can arise about indications to use this approach to remove pulmonary metastases. Moreover, the role of lymphadenectomy remains unclear although some authors believe that radical lymph node dissection can add objective benefit to overall survival; but this view is again not supported by scientific evidence [25].

In this situation of uncertainty, the gold standard surgical treatment cannot be offered to our patients, and the surgical indications together with the choice of the surgical approach still depends more on the single center or surgeon practice than on a basis of strong scientific evidence. Moreover, studies reporting survival rates of $30-50 \%$ analyze patient selected on the basis of good prognostic features and this represents a bias that can influence survival results [26]. This uncertainty is due to the lack of prospective studies.

Returning to our main question: in case of uncertainly, what is the best approach? VATS or TT? It is obvious that in absence of proved long-term survival with TT, the less invasive approach should be used, and therefore VATS should be preferred. Moreover, VATS is also a good approach to perform lymphadenectomy. However in our center we perform an 'hybrid' approach that utilizes a VATS and minithoracotomy that permits the entire hand to palpate the lung. We have used this approach in 14 patients, and 34 wedges resection were performed with hospital stay of 4 days (2-9 days).

In March 2010 it has been started [27] the PM for Colorectal Cancer trial (PulMiCC), a prospective multicenter randomized trial with the primary goal to establish the effective benefit of performing PM in patients with pulmonary metastasis of colorectal cancer. The trial is opened internationally with centers in Italy and Serbia

\begin{tabular}{|c|c|c|c|c|c|}
\hline Year & Pts (n) & $\begin{array}{l}\text { Missed } \\
\text { nodules (\%) }\end{array}$ & $\begin{array}{l}\text { Missed } \\
\text { metastases (\%) }\end{array}$ & $\begin{array}{l}\text { Missed } \\
\text { malignancy (\%) }\end{array}$ & Author \\
\hline 2014 & 215 & 36 & & 48 & Althagafi et al. [15] \\
\hline 2009 & 57 & 37 & 18 & & Cerfolio et al. [16] \\
\hline 2011 & 152 & 34 & & 20 & Cerfolio et al. [2] \\
\hline 2004 & 16 & 31 & & & $\begin{array}{l}\text { Detterbeck and } \\
\text { Egan [17] }\end{array}$ \\
\hline 2014 & 89 & 41 & 33 & 36 & Eckardt and Licht [18] \\
\hline 1996 & 18 & 78 & & 56 & McCormack et al. [19] \\
\hline 2009 & 52 & 2 & & & Nakas [13] \\
\hline
\end{tabular}


[28,29]. Although the aim is to establish which patients take benefit from PM, between the large amount of prospective data of the PulMiCC trial will hopefully provide conclusive evidence as to whether the use of TT prolong survival in lung metastasis.

\section{Conclusion}

Our study confirms that overall survival is equivalent for VATS and TT, therefore the 'gold standard' surgical treatment for lung metastases remains a point of debate. Evidence is weak to suggest that VATS is better than TT or vice versa. The consensus is far to be decided as there are no prospective randomized trial that could clarify the question. Surgeons will continue to operate according to their practice until scientific evidence will prove what the best approach is for LM.

\section{Financial \& competing interests disclosure}

The authors have no relevant affiliations or financial involvement with any organization or entity with a financial interest in or financial conflict with the subject matter or materials discussed in the manuscript. This includes employment, consultancies, honoraria, stock ownership or options, expert testimony, grants or patents received or pending or royalties.

No writing assistance was utilized in the production of this manuscript.

\section{References}

1 Van Raemdonk D, Friedel G. The European Society of Thoracic Surgeons lung metastasectomy project. J. Thorac. Oncol. 5, 127-129 (2010).

2 Cerfolio RJ, Bryant AS, McCarty TP, Minnich DJ. A prospective study to determine the incidence of non-imaged malignant pulmonary nodules in patients who undergo metastasectomy by thoracotomy with lung palpation. Ann. Thorac. Surg. 91, 1696-1700; Discussion 1700-1691 (2011).

3 Althagafi KT, Alashgar OA, Almaghrabi HS et al. Missed pulmonary metastasis. Asian Cardiovasc. Thorac. Ann. 22(2), 183-186 (2014).

4 Dong S, Zhang L, Li W, Du J, Liu X, Chen $\mathrm{X}$. Evaluation of VATS for pulmonary metastases: a meta-analysis. PLoS ONE 9(1), e85329 (2014).

5 Herle P, Cheang M, Antippa P. Pulmonary metastasectomy by VATS or open thoracotomy: a systematic review and meta-analysis of long term outcomes. J. Cardiothorac. Surg. 8(Suppl. 1), Abstract O242 (2013).

6 Greenwod A, West D. Is a thoracotomy rather than thoracoscopic resection associated with improved survival after pulmonary metastasectomy? Interact. Cardiovasc. Thorac. Surg. 17, 720-724 (2013)

7 Molnar TF, Gebitekin C, Turna A. What are the considerations in the surgical approach in pulmonary metastasectomy? J. Thorac. Oncol. 5, S140-S144 (2010).

8 Carballo M, Maish MS, Jaroszewski DE, Holmes CE. Video-assisted thoracic surgery (VATS) as a safe alternative for the resection of pulmonary metastases: a retrospective cohort study. J. Cardiothorac. Surg. 4, 13 (2009).

9 Chao YK, Chang HC, Wu YC et al. Management of lung metastases from colorectal cancer: video-assisted thoracoscopic surgery versus thoracotomy - a case matched study. Thorac. Cardiovasc. Surg. 60(6), 398-404 (2012).

10 Gossot D, Radu C, Girard P et al. Resection of pulmonary metastases from sarcoma: can some patient from a less invasive approach? Ann. Thorac. Surg. 87(1), 238-243 (2009).

11 Mutsaerts EL, Zoetmulder FA, Meijer S et al. Long-term survival of thoracoscopic metastasectomy vs metastasectomy by thoracotomy in patients with a solitary pulmonary lesion. Eur. J. Surg. Oncol. 28, 864-868 (2002).

12 Nakajima J, Murakawa T, Fukami T, Takamoto $S$. Is thoracoscopic surgery justified to treat pulmonary metastasis from colorectal cancer? Interact. Cardiovasc. Thorac. Surg. 7 , 212-217 (2008).

13 Nakas A, Klimatsidas MN, Entwisle J et al. Video-assisted versus open pulmonary metastasectomy: the surgeon's finger or the radiologist's eye? Eur. J. Cardiothorac. Surg. 36, 469-474 (2009).

14 Watanabe M, Deguchi H, Sato M et al. Midterm results of thoracoscopic surgery for pulmonary metastases especially colorectal cancers. J. Laparoendosc. Adv. Surg. Tech. 8, 195-200 (1998).

15 Althagafi KT, Alashgar OA, Almaghrabi HS et al. Missed pulmonary metastases. Asian Cardiovasc. Thorac. Ann. 22(2), 183-186 (2012).

16 Cerfolio RJ, McCarty T, Bryant AS. Non-imaged pulmonary nodules discovered during thoracotomy for metastasectomy by lung palpation. Eur. J. Cardiothorac. Surg. 35, 786-791 (2009).

17 Detterbeck F, Egan TM. Thoracoscopy using a substernal handport for palpation. Ann. Thorac. Surg. 78, 1031-1036 (2004).

18 Eckardt J, Licht PB. Thoracoscopic or open surgery for pulmonary metastasectomy: an observer blinded study. Ann. Thorac. Surg. 98(2), 466-470 (2014).

19 McCormack PM, Bains MS, Beg CB et al. Role of videoassisted thoracic surgery in the treatment of pulmonary metastases: results of a prospective trial. Ann. Thorac. Surg. 62, 213-216 (1996).

20 Rush VW. Pulmonary metastasectomy. Current indications. Chest 107, 322-331 (1995).

21 Friedel G, Pastorino U, Buyse M et al. Resection of lung metastases: long-term results and prognostic analysis based on 5206 cases - The International Registry of Lung Metastases. Zentralbl. Chir. 124, 96-103 (1999).

22 Ripley RT, Downey RJ. Pulmonary metastasectomy. J. Surg. Oncol. $109,42-46$ (2014).

23 Rocco G, Martucci N, La Manna $\mathrm{C}$ et al. et al. Ten-year experience on 644 patients undergoing single-port (uniportal) video-assisted thoracoscopic surgery. Ann. Thorac. Surg. 96(2), 434-438 (2013).

24 Mineo T C, Pompeo E, Ambrogi V, Pistolese C. Video-assisted approach for transxiphoid bilateral lung metastasectomy. Ann. Thorac. Surg. 67(6), 1808-1810 (1999).

25 Renaud S, Alifano M, Falcoz PE et al. does nodal status influence survival? Results of a 19 year systematic lymphadenectomy 
experience during lung metastasectomy of colorectal cancer. Inter. Cardiovasc. Thorac. Surg. 18(4), 1-6 (2014).

26 Treasure T, Milosevic M, Fiorentino F, Macbeth F. Pulmonary metastasectomy: what is the practice and where is the evidence for effectiveness? Thorax doi:10.1136/thoraxjnl2013-204528 (2014) (Epub ahead of print).

27 Treasure T, Fallowfield L, Lees B. Pulmonary metastasectomy in colorectal cancer: the PulMiCC trial. J. Thorac. Oncol. 5, 203-206 (2010).
28 Treasure T, Miloševic M, Migliore M, Lees B. Pulmonary Metastasectomy in Colorectal Cancer (PulMiCC International). Colorectal Cancer 2(6), 505-513 (2013).

29 Migliore M, Milosevic M, Lees B, Treasure T. The PulMiCC is open in Italy. Oncologist 18(5), 637 (2013). 